\title{
PROPERTIES OF NONTURBULENT ROUND LIQUID JETS IN UNIFORM CROSSFLOWS
}

\author{
C. Aalburg, K.A. Sallam, G.M. Faeth ${ }^{*}$ \\ The University of Michigan, Ann Arbor, MI 48109-2140
}

\begin{abstract}
A computational and experimental study of the deformation and breakup properties of nonturbulent round liquid jets in uniform gaseous crossflows is described, seeking to develop numerical predictions to find these properties at conditions that are difficult to address using experiments. The time-dependent incompressible two-dimensional Navier-Stokes equations were solved in the gas and liquid phases in conjunction with the level-set method to determine the position of the liquid/gas interface of the deforming liquid jets. The computations were evaluated satisfactorily based on earlier measurements for solid circular cylinders in crossflow (recirculating wake lengths, drag coefficients, conditions for the onset of eddy shedding, and frequencies of eddy shedding) and present measurements of the properties of nonturbulent round liquid jets in crossflow (liquid jet crosstream deformation, liquid jet streamwise deformation and deflection, and breakup regime transitions). Subsequent computations to find liquid jet deformation and breakup properties revealed relatively small effects of liquid/gas density ratios on deformation and breakup regime boundaries. Small Reynolds number conditions approaching the Stokes flow regime, however, resulted in a significant increase of the resistance of liquid jets in crossflow
\end{abstract}

\footnotetext{
* A.B. Modine Professor, Department of Aerospace Engineering, Fellow AIAA, Corresponding author, Tel.: +1-734-764-7202; Fax: +1-734-936-0106; E.mail: gmfaeth@umich.edu (G.M. Faeth).
}

to deformation that has not been addressed by existing measurements of breakup properties.

\section{NOMENCLATURE}

$\mathrm{C}_{\mathrm{D}} \quad$ drag coefficient

$d_{c} \quad$ cross stream dimension of liquid column

$d_{s} \quad$ streamwise dimension of liquid column

$\mathrm{d}_{\mathrm{o}} \quad$ initial diameter of liquid column

$\mathrm{f} \quad$ eddy-shedding frequency

$\mathrm{L} \quad$ length of wake behind a solid cylinder

Oh Ohnesorge number, $\mu_{\mathrm{L}} /\left(\rho_{\mathrm{L}} \mathrm{d}_{\mathrm{o}} \sigma\right)^{1 / 2}$

q liquid/gas momentum ratio, $\rho_{\mathrm{L}} \mathrm{U}_{\mathrm{j}}^{2} /\left(\rho_{\mathrm{G}} \mathrm{U}_{\mathrm{o}}^{2}\right)$

$\operatorname{Re} \quad$ jet Reynolds number, $\rho_{\mathrm{G}} \mathrm{U}_{\mathrm{o}} \mathrm{d}_{\mathrm{o}} / \mu_{\mathrm{G}}$

St Strouhal number, $\mathrm{fd}_{\mathrm{o}} / \mathrm{U}_{\mathrm{o}}$

$\mathrm{t}$ time

$\mathrm{t}^{*} \quad$ characteristic breakup time, $\left(\rho_{\mathrm{L}} / \rho_{\mathrm{G}}\right)^{1 / 2} \mathrm{~d}_{\mathrm{o}} / \mathrm{U}_{\mathrm{o}}$

$\mathrm{U}_{\mathrm{j}} \quad$ jet exit velocity

$\mathrm{U}_{\mathrm{o}} \quad$ crossflow velocity

We Weber number, $\rho_{\mathrm{G}} \mathrm{d}_{\mathrm{o}} \mathrm{U}_{\mathrm{o}}{ }^{2} / \sigma$

$\mathrm{x} \quad$ distance in the direction of the crossflow

$\mathrm{y} \quad$ distance in the direction of the liquid jet

$\mu \quad$ molecular viscosity

$\rho \quad$ density

$\sigma \quad$ surface tension

\section{Subscripts}

G gas phase property

L liquid phase property

\section{INTRODUCTION}

The deformation and breakup properties of nonturbulent round liquid jets in uniform nonturbulent crossflows were studied computationally and experimentally. The study is 
motivated by applications to the primary breakup of liquid jets in crossflow that are encountered in airbreathing propulsion systems, liquid rocket engines, diesel engines, spark ignition engines, and agricultural sprays, among others. Due to experimental constraints, past experimental studies of the primary breakup properties of nonturbulent round liquid jets in gaseous crossflows by Vich, ${ }^{1}$ Mazallon et al., ${ }^{2}$ Sallam et al. ${ }^{3}$ and $\mathrm{Wu}$ et al. ${ }^{4}$ were limited to large liquid/gas density ratios and relatively small Ohnesorge numbers. The objective of the present study was to use numerical predictions in order to consider the moderate liquid/gas density ratios and large Ohnesorge number conditions that are more representative of practical high-pressure spray combustion applications.

Numerous past experimental and computational studies of round liquid jets in gaseous crossflows have emphasized penetration lengths and jet/spray plume trajectories. ${ }^{5-16}$ The deformation and primary breakup processes at the liquid surface have recently received more attention, however, with several investigators ${ }^{1-4}$ reporting striking similarities between the breakup of round liquid jets in crossflow and the secondary breakup of drops subjected to shock wave disturbances. ${ }^{17-29}$ In particular, analogous to the secondary breakup of drops, the primary breakup of round liquid jets displayed regimes of bag, multimode and shear breakup whose transitions were governed by the Weber number. Liquid jet velocities were found to have little effect, i.e., variations of the liquid/gas momentum ratio, q, in the range 3-8000 had no effect on liquid jet deformation and breakup properties., ${ }^{2,3}$ Existing information about the primary breakup of liquid jets in crossflows, however, is limited to relatively large liquid/gas density ratios (in excess of 500) and relatively small Ohnesorge numbers (smaller than 0.12), providing motivation to use computational methods in order to investigate parameter ranges that are difficult to address using experiments.

In view of these observations, the objective of the present investigation was to develop a computational method to investigate the deformation and primary breakup properties of nonturbulent round liquid jets in uniform crossflows, as follows: formulate the computational method, use existing measurements of the properties of solid cylinders in uniform crossflow to evaluate the numerical predictions, use both existing and new measurements of the deformation and deflection properties of nonturbulent round liquid jets in crossflow to evaluate the predictions, and finally to exploit the numerical predictions to study the properties of sprays at the small liquid/gas density ratios and large Ohnesorge number conditions of interest for practical high pressure combustion processes.

\section{COMPUTATIONAL METHODS}

The measurements of Mazallon et al. ${ }^{2}$ and Sallam et al. $^{3}$ showed that the deformation and breakup properties of round nonturbulent liquid jets in uniform crossflows were independent of liquid jet velocities which implies that the various streamwise planes of the liquid jet do not interact. This assumption was adopted during the present study so that liquid column behavior is taken to be equivalent to the temporal behavior of an initially motionless two-dimensional cylindrical liquid jet element subjected to a step increase of the ambient crossflow velocity. It was further assumed that effects of evaporation are small, liquid and gas phase properties are constant, the liquid jet velocity remains constant at its initial value, $U_{j}$, and the liquid jet flow and the crossflow are nonturbulent. Notably, all these assumptions correspond to the experimental conditions of Mazallon et al., ${ }^{2}$ Sallam et al. ${ }^{3}$ and the present investigation that will be used to evaluate the present numerical predictions. Under the present approximations, the distance traversed by the cylindrical jet element in the initial jet direction, $\mathrm{y}$, is therefore the product of the constant jet velocity, $\mathrm{U}_{\mathrm{j}}$, and the time of interaction between the liquid jet and the crossflow, t:

$$
\mathrm{y}=\mathrm{U}_{\mathrm{j}} \mathrm{t}
$$

Other major assumptions of the analysis were as follows: normal and tangential forces are continuous across the liquid surface, i.e., the surface is infinitely thin and has negligible mass storage and inertia capabilities; the surface tension is constant; effects of buoyancy are negligible; flow Mach numbers are small so that effects of viscous dissipation and kinetic energy can be neglected; and the flow is isothermal. Effects of eddy shedding were considered at appropriate operating conditions. These approximations represent a model of liquid breakup conditions that has been reasonably effective during evaluation of predictions for the properties of secondary breakup of drops subjected to shock wave disturbances by Aalburg et al. ${ }^{30}$ 


\section{Numerical Methods}

The time-dependent and two-dimensional Navier-Stokes equations were solved in both gas and liquid phases using the projection method of Chorin. ${ }^{31}$ The discretization in space was carried out on a staggered grid according to the incompressible marker and cell (MAC) method of Harlow and Welch. ${ }^{32}$ The liquid/gas interface was captured by the level-set method of Sussman et al.;3 this approach yielded the local fluid properties of each cell with a smooth transition between the gas and the liquid phases near the interface. A redistancing algorithm due to Sussman and Fatemi ${ }^{34}$ was used to maintain the level-set as an accurate distance function at all times. The interface calculations allowed for effects of surface tension, pressure and shear forces, with surface tension represented by a body force distributed over an interface having finite thickness, following Brackbill et al. $^{35}$ The discretizations in both space and time were secondorder accurate.

When eddy shedding was absent, the domain size was $5 \mathrm{~d}_{\mathrm{o}}$ wide and $12.5 \mathrm{~d}_{\mathrm{o}}$ long and was covered with a moving grid that was 256-512 elements wide and 640-1280 elements long. The boundary conditions were symmetric along the sides of the computational domain with a constant fluid velocity across the inlet and a fixed pressure along the outlet. When eddy shedding was present, the domain width and the number of elements were doubled with no assumption of symmetry of the flow over the liquid jet. Tests of various sized solution regions and finer grids caused less than a $2.5 \%$ change of computational results reported in the following; therefore, computational errors are conservatively estimated to be smaller than $4 \%$.

\section{Computational Conditions}

The ranges of present calculations were as follows: Reynolds numbers of 12.5-200, Weber numbers of $0.1-100,000$, Ohnesorge numbers of $0.001-100$, liquid/gas density ratios of $2-\infty$ and liquid/gas molecular viscosity ratios of 0.001-1,000.

\section{EXPERIMENTAL METHODS}

$\underline{\text { Apparatus }}$

The experiments were carried out inside a subsonic wind tunnel with a cross section of 610 $\times 610 \mathrm{~mm}$ with windowed side walls to provide optical access to observe the trajectories and the deformation and breakup properties of nonturbulent round liquid jets at uniform crossflow velocities of 5$18 \mathrm{~m} / \mathrm{s}$ at normal temperature and pressure (see Sallam et al. ${ }^{3}$ ).

Using a pressure feed system capable of injection pressures up to $1.5 \mathrm{MPa}$, the liquid jets were injected vertically downward through round supercavitating nozzles which had sharp-edged inlets and exits with passage length-to-diameter ratios smaller than 3 to create nonturbulent round liquid jets with no tendency to break up in the absence of crossflow (for jet Reynolds numbers as large as 30,000).

\section{Instrumentation}

Present measurements were confined to liquid jet deformation and deflection prior to the onset of ligament and drop formation along the liquid jet; therefore, conditions were excellent for flow visualization and measurements could be made using pulsed shadowgraphy. A frequency-doubled YAG laser (Spectra Physics, Model GCR-130, 532 $\mathrm{nm}$ wavelength, $7 \mathrm{~ns}$ pulse duration and up to 300 $\mathrm{mJ}$ optical energy per pulse) was used for the light source of the shadowgraph system. Deformation of the liquid jets in the streamwise direction of the crossflow, the onset of breakup, and liquid jet trajectories were obtained from these shadowgraphs with experimental uncertainties (95\% confidence) smaller than $10 \%$.

\section{Test Conditions}

Test conditions involved two different liquids (water and ethyl alcohol), liquid injector diameters of 0.5 and $1.0 \mathrm{~mm}$, liquid jet velocities of $9-14 \mathrm{~m} / \mathrm{s}$ and air crossflow velocities of $5-18 \mathrm{~m} / \mathrm{s}$. This yielded the following ranges of normalized test variables: liquid/gas density ratios of 806 and 997, liquid/gas viscosity ratios of 48 and 66, crossflow Reynolds numbers of 345-1100, crossflow Weber numbers of $0.6-12$, liquid/gas momentum ratios of 170-6700, and jet Ohnesorge numbers of 0.0030.013. Crossflow Mach numbers were smaller than 0.1 ; therefore, compressibility effects were negligible.

\section{RESULTS AND DISCUSSION}

Computational Evaluation

The numerical predictions were evaluated based on earlier measurements and computations (predictions) of the properties of crossflows over 
solid cylinders. These predictions were carried out considering liquid jets having very large densities and viscosities so that the liquid jet did not deflect or deform or internally recirculate significantly as a result of the crossflow.

Typical predictions of streamline patterns for crossflow over solid cylinders are illustrated in Fig. 1. The streamline patterns are illustrated for two Reynolds numbers, $\mathrm{Re}=30$ and 170 , at times $\mathrm{tU}_{\mathrm{o}} / \mathrm{d}_{\mathrm{o}}=$ 10 and 40 after the start of crossflow, respectively. At $R e=30$ (top picture of Fig. 1) a stable wake is present on the downstream side of the cylinder, consisting of a symmetric pair of elongated vortices. Predictions of the temporal development of these stable wakes as well as their finals lengths were compared with measurements of Coutanceau and Bouard $^{36}$ and the computations of Kawaguti and Jain $^{37}$ and Thoman and Szewczyk, ${ }^{38}$ showing good agreement within the uncertainties of the results. At $\mathrm{Re}=170$ (bottom picture of Fig. 1) eddy shedding is present and the flow over the cylinder is no longer symmetric, and does not approach a steady-state condition, so that the full width of the flow must be considered. In spite of the somewhat irregular flow patterns, eddy shedding proved to be relatively periodic and yielded a constant eddy-shedding frequency.

Measurements and computations of eddyshedding frequencies for crossflow over solid cylinders are illustrated in Fig. 2. Eddy-shedding frequencies, f, represented by the Strouhal number, $\mathrm{St}=\mathrm{fd}_{\mathrm{o}} / \mathrm{U}_{\mathrm{o}}$, are plotted as a function of the Reynolds number of the crossflow, with the present predicted onset of eddy shedding at $\mathrm{Re} \approx 40$ also marked on the plot. Results shown on the figure include the measurements of Kovasznay, ${ }^{39}$ Roshko, ${ }^{40}$ Berger and Wille $^{41}$ and Nishioka and Sato. ${ }^{42}$ The agreement among all the measurements and predictions is excellent. Notably, this agreement also extends to the determination of the onset of eddy shedding for all these results at $\mathrm{Re} \approx 40$.

The final parameter that was considered during evaluation of the computations was the drag coefficient, $\mathrm{C}_{\mathrm{D}}$, of a solid cylinder in crossflow. Present predictions for the drag coefficient for crossflow Reynolds numbers of 5-200 showed good agreement with the measurements of Wieselsberger, ${ }^{43}$ Tritton $^{44}$ and Nishioka ${ }^{45}$ and the computed results of Takami and Keller ${ }^{46}$ and Dennis and Chang. ${ }^{47}$ Taken together, the agreement between present predictions and earlier results for flows normal to solid cylinders was satisfactory; therefore, subsequent computations addressed the behavior of round liquid jets in crossflow which was the main objective of the present investigation.

\section{Flow Visualization}

The properties of round liquid jets in uniform crossflow for a given initial diameter are affected by the Reynolds number, the Weber number, the Ohnesorge number, and the liquid/gas density ratio. In the following, the shape of the interface of a round liquid jet as a function of time after exposure to a step change in cross stream velocity will be considered for the last three variables - Weber number, Ohnesorge number and liquid/gas density ratio - while keeping the Reynolds number constant at $\mathrm{Re}=50$ because it has a smaller effect on the flow than the other parameters for $\operatorname{Re} \geq 50$. In each case, ambient flow is from left to right and the shape of the interface will be shown at $\mathrm{t} / \mathrm{t}^{*}=0,1,2, \ldots, 6$, where $t^{*}=\left(\rho_{\mathrm{L}} / \rho_{\mathrm{G}}\right)^{1 / 2} \mathrm{~d}_{\mathrm{o}} / \mathrm{U}_{\mathrm{o}}$ is the characteristic breakup time of round liquid drops at small Ohnesorge numbers defined by Ranger and Nicholls. ${ }^{29}$ The left-most contour is the initial condition.

Figure 3 shows the influence of the Weber number on the shape of the interface of round liquid jets in uniform crossflows for $\mathrm{We}=1,4$ and 8 at $\mathrm{Re}=50, \rho_{\mathrm{L}} / \rho_{\mathrm{G}}=2$, and $\mathrm{Oh}=0.01$. The liquid column is seen to respond to the ambient fluid flow by both translating in the streamwise direction (i.e., as a liquid jet this would involve following a trajectory that is deflected in the streamwise direction) and deforming from its initial circular shape. The translation is caused by the drag of the ambient flow on the liquid column as a whole. The shape deformation comes about because acceleration of the ambient fluid over the liquid column yields reduced pressures at its sides which draws the liquid in the cross stream direction until it is eventually brought to a stop by counteracting surface tension forces which tend to bring the flow back to its initial state in a decaying periodic manner as acceleration of the liquid eventually relaxes the velocity difference between the liquid column and the ambient fluid to a negligible value. Increasing Weber number implies decreasing surface tension, which results in progressively larger lateral deformation of the liquid column with also leads to larger drag forces and drag coefficients. The corresponding reduced liquid column stability and increased drag forces eventually lead to breakup of the liquid column, but at 
somewhat larger values of We than considered in Fig. 3. This behavior is remarkably similar to processes of deformation and secondary breakup of round liquid drops exposed to shock wave disturbances, see Chou and Faeth, ${ }^{19}$ Chou et al., ${ }^{20}$ Dai and Faeth, ${ }^{21}$ Faeth et al., ${ }^{22}$ Hsiang and Faeth, ${ }^{23-25}$ and references cited therein.

Figure 4 shows the influence of the Ohnesorge number on the shape of the interface of round liquid jets in uniform crossflows for $\mathrm{Oh}=0.01$, 0.1 and 1.0 at $\mathrm{Re}=50, \rho_{\mathrm{L}} / \rho_{\mathrm{G}}=2$, and $\mathrm{We}=8$. Conditions for the lowermost illustration in Fig. 4 are identical to those for the uppermost illustration in Fig. 3. Then increasing Ohnesorge numbers from this condition, implying increasing liquid viscosity, tends to slow the rate of lateral deformation of the liquid column so that maximum deformation is reached when the velocity differences between the liquid jet and the ambient fluid are reduced; this correspondingly reduces the pressure drop from the forward stagnation point to the side of the liquid column and thus the maximum degree of deformation for a given Weber number. Another effect of increasing Ohnesorge number is to inhibit oscillations of the liquid jet, eventually eliminating the oscillatory deformation regime at Ohnesorge numbers somewhat larger than those considered in Fig. 5

Figure 5 shows the influence of the liquid/gas density ratio on the shape of the interface of round liquid jets in uniform crossflows for $\rho_{\mathrm{L}} / \rho_{\mathrm{G}}=2,8$ and 32 at $\mathrm{Re}=50, \mathrm{We}=4$, and $\mathrm{Oh}=0.01$. Conditions for the lowermost illustration in Fig. 5 are identical to those of the middle illustration in Fig. 3. Then increasing the liquid/gas density ratio implies increasing the relaxation time $\left(t^{*} \sim\left(\rho_{\mathrm{L}} / \rho_{\mathrm{G}}\right)^{1 / 2}\right)$ whereas decreasing jet acceleration in the transverse direction which scales with $\left(\rho_{\mathrm{L}} / \rho_{\mathrm{G}}\right)^{-1}$. The characteristic breakup time of Ranger and Nicholls ${ }^{29}$ tends to scale liquid/gas inertial effects on deformation and breakup times correctly, however, so that the nature of the liquid column contour tends to remain qualitatively similar for similar dimensionless times, $\mathrm{t} / \mathrm{t}^{*}$, for the results illustrated in Fig. 5. Therefore, jet velocities relative to the gas phase are proportional to $\left(\rho_{\mathrm{L}} / \rho_{\mathrm{G}}\right)^{-1 / 2}$. Increasing the liquid/gas density ratio then implies a larger relative velocity between the jet and the crossflow over the time of deformation, explaining the progressively larger transverse distances traveled by the jet for each $\mathrm{t} / \mathrm{t}^{*}$ time increment as the liquid/gas density ratio increases.

\section{Liquid Jet Deformation}

The deformation properties of initially round liquid jets in crossflow are readily seen by the plots of normalized deformation, $\mathrm{d}_{\mathrm{c}} / \mathrm{d}_{\mathrm{o}}$, as a function of normalized time, $\mathrm{t} / \mathrm{t}^{*}$, illustrated in Fig. 6. The property $d_{c}$ is the maximum cross stream dimension of the liquid column at any given time. The conditions considered in Fig. 6 include those considered in the visualizations of Figs. 3-5, but are somewhat expanded to include We of 1-16, Oh of $0.001-1.0$ and $\rho_{\mathrm{L}} / \rho_{\mathrm{G}}$ of $2-32$.

The uppermost illustration in Fig. 6 displays effects of varying the Weber number for $\rho_{\mathrm{L}} / \rho_{\mathrm{G}}=2$ and $\mathrm{Oh}=0.01$. The maximum degree of deformation progressively increases as the Weber number increases, eventually exceeding $d_{c} / d_{o}=2$ for We in the range 8-16, where breakup of liquid jets in crossflow, and round liquid drops subjected to shock wave disturbances, generally is observed, see Refs. 2 and 3. For conditions prior to breakup, $d_{c} / d_{o}<2$ and We $\leq 8$, the column shape oscillates during relaxation back to a round jet configuration with the period of oscillation progressively increasing as the Weber number is increased.

The middle illustration of Fig. 6 displays effects of varying the Ohnesorge number for $\rho_{\mathrm{L}} / \rho_{\mathrm{G}}=2$ and $\mathrm{We}=8$. For values of $\mathrm{Oh} \leq 0.01$, varying $\mathrm{Oh}$ has little effect on either the amplitude or the frequency of oscillatory deformation. Increasing the Ohnesorge number beyond 0.01 , however, causes a progressive decrease of the maximum amplitude of deformation, and eventually leads to a nonoscillatory relaxation process for $\mathrm{Oh} \geq 1.0$, by exceeding the critically damped condition for this process. This behavior is similar to the effect of $\mathrm{Oh}$ on secondary drop breakup where increasing $\mathrm{Oh}$ also leads to the termination of the oscillatory deformation regime. ${ }^{22-24}$

The bottom illustration of Fig. 6 displays effects of varying the liquid/gas density ratio for $\mathrm{We}=4$ and $\mathrm{Oh}=0.01$. It is evident that increasing the liquid/gas density ratio tends to increase maximum levels of deformation for given values of We and $\mathrm{Oh}$, caused by the progressively larger relative velocities between jet and crossflow as the density ratio is increased, as already discussed in connection with Fig. 5. Normalizing the time with the characteristic Ranger and Nicholls ${ }^{29}$ breakup time, however, helps 
account for effects of liquid inertia on liquid column oscillations so that the frequency of oscillation in terms of $t / t^{*}$ tends to be relatively independent of the liquid/gas density ratios.

Finally, in order to further evaluate the computational predictions, present numerical results for liquid jet deformation were compared with present experimental measurements of the streamwise deformation of round liquid jets subjected to uniform crossflow in a wind tunnel. This required computing liquid column response to crossflows for relatively large density ratios, e.g., $\rho_{\mathrm{L}} / \rho_{\mathrm{G}}=700-1100$. Achieving accurate computational results at such conditions is problematical, however, because the increased $\mathrm{t}^{*}$ requires proportionally longer integration times. In order to avoid this difficulty, computations were carried out at reasonably tractable liquid/gas density ratios $\left(\rho_{\mathrm{L}} / \rho_{\mathrm{G}}=2-256\right)$ and Richardson extrapolation was used to find results at large liquid/gas density ratios for evaluation using the present measurements.

Measured and predicted temporal development of the deformation of round liquid jets in crossflow are illustrated in Fig. 7, considering water and ethyl alcohol jets ejecting from 0.5 and 1.0 $\mathrm{mm}$ diameter nozzles. The deformation is represented by $d_{s} / d_{o}$ where $d_{s}$ is the maximum streamwise dimension of the liquid column which is an easily observable property of the liquid jet. Similar to the results illustrated in Fig. 6, times are normalized with the Ranger and Nicholls ${ }^{29}$ characteristic time for a liquid column. Results are illustrated for values of the Weber number in the range 1.75-32. For $\mathrm{We}=14-32$, primary breakup occurs along the surface of the jet after a time; therefore, measurements and predictions are terminated when this condition is reached. In general, the agreement between measurements and predictions in Fig. 7 is good, well within the range anticipated in view of experimental uncertainties and computational accuracy.

\section{$\underline{\text { Liquid Jet Deflection }}$}

Measured and predicted deflections of round liquid jets in crossflow are illustrated in Fig. 8. The deflection is represented by the crosstream distance, $x$, traversed by the axis of the liquid jet from its initial position after a given time period of exposure to the crossflow, t. The experimental results were obtained for relatively small Weber numbers in the range 0.6-2.7 and Ohnesorge numbers in the range $0.003-0.01$; this places the jet in the column breakup regime where it is deflected appreciable distances downstream and no breakup occurs along the liquid surface, providing an extended region for testing present predictions. The conditions of the predictions generally match those of the measurements. It is evident that the scatter of the measurements is consistent with the range of We considered and experimental uncertainties; in addition, predictions and measurements agree within similar bounds.

\section{Deformation Regime Maps}

Figure 9 is the deformation and breakup regime map for liquid jets in crossflow in the classical $\mathrm{Hinze}^{23}$ approach, which has the deformation and breakup properties plotted as a function of the liquid jet drag-force/surface-tensionforce ratio (represented by the Weber number) vs. the liquid jet viscous-force/surface-tension-force ratio (represented by the Ohnesorge number). The computations provide normalized maximum liquid jet deformation values of $\left(\mathrm{d}_{\mathrm{cmax}}-\mathrm{d}_{\mathrm{o}}\right) / \mathrm{d}_{\mathrm{o}}=50$ and $100 \%$, whereas, the measurements provide maximum normalized deformations at $50 \%$ and the boundary for the onset of breakup. The experimental conditions of the measurements and numerical predictions are comparable. Measured and predicted maximum jet deformations of $50 \%$ are in excellent agreement. The results in Fig. 9 also show that the predicted normalized maximum deformation of $100 \%$ provides an excellent estimate of the conditions required for the onset of breakup. This behavior is very similar to the relationship between a computed normalized maximum deformation of $80 \%$ and measured onset of secondary drop breakup for drops subjected to shock wave disturbances observed by Aalburg et al. ${ }^{30}$ Thus a predicted breakup approximation for liquid jets at a maximum normalized deformation of $100 \%$ will be adopted in the following as a simplified way to obtain some insight about effects of liquid gas density ratio, etc., on liquid jet breakup conditions using computations of deformation.

As discussed by Aalburg et al. ${ }^{30}$ and Sallam et al., ${ }^{3}$ it is easily shown that Weber and Ohnesorge numbers govern breakup regime transitions for the liquid jets in crossflow (the effect of other properties on breakup regime transitions will be discussed subsequently): for conditions when viscous forces are small, gas dynamic forces (drag) on the liquid jet must be stabilized by surface-tension-forces, which implies that deformation and breakup regime 
transitions correspond to particular critical Weber numbers, i.e., $\mathrm{We}_{\mathrm{cr}}=$ const.; whereas, for conditions where surface tension forces are small, gas dynamic forces (drag) on the jet must be stabilized by liquidviscous forces which implies that critical Weber numbers are proportional to the square of the Ohnesorge number, i.e., $\mathrm{We}_{\mathrm{cr}} \sim \mathrm{Oh}^{2}$. It is evident that the results illustrated in Fig. 9 generally satisfy these requirements: for $\mathrm{Oh}<1$, regime transitions are independent of $\mathrm{Oh}$ and are given by constant values of $\mathrm{We}_{\mathrm{cr}}$; in contrast, for $\mathrm{Oh}>>1$, predicted results indicate that $\mathrm{We}_{\mathrm{cr}} \sim \mathrm{Oh}^{2}$ as expected.

A better approach for representing conditions where liquid viscosity effects are important (at large $\mathrm{Oh}$ conditions), developed by Aalburg et al. ${ }^{30}$ for the secondary breakup of drops, is to account for liquid viscous effects directly by plotting the ratio of drag to liquid viscous forces, $\mathrm{We}^{1 / 2} / \mathrm{Oh}$, as the ordinate of the plot, rather than the ratio of drag to surface tension forces, We (for a region where surface tension is less important than liquid viscous forces). Based on the ideas of Aalburg et al. $^{30}$, the deformation and breakup boundaries of Fig. 9 were plotted in the new $\mathrm{We}^{1 / 2} / \mathrm{Oh}$ vs. $1 / \mathrm{Oh}$ coordinates in Fig. 10 to provide a deformation and breakup regime map that is complimentary to Hinze's traditional approach (We vs. Oh) but more relevant to the large Ohnesorge number conditions that are often encountered in practical high-pressure spray combustion applications. The measured breakup boundaries for large values of $1 / \mathrm{Oh}$ due to Mazallon et al. ${ }^{2}$ and Sallam et al. ${ }^{3}$ are in good agreement with the numerical predictions for a maximum normalized liquid jet deformation of $100 \%$, as expected based on the results of Fig. 9. At small values of $1 / \mathrm{Oh}$ (large $\mathrm{Oh}$ conditions), deformation and breakup boundaries are now constant in terms of $\mathrm{We}^{1 / 2} / \mathrm{Oh}$. In addition, the results illustrate that effects of liquid/gas density ratio are relatively small for values in the range 32$\infty$, particularly for conditions where $1 / \mathrm{Oh}$ is large.

Finally, effects of Reynolds number on the deformation and breakup regime boundaries of liquid jets in crossflow were found to be small for Re>50 similar to past observations for the secondary breakup of drops. ${ }^{30}$ This behavior is illustrated by the results plotted in Fig. 11, where the Weber number for a maximum normalized liquid jet deformation of $100 \%$ is plotted as a function of Reynolds number for $\mathrm{Oh}=0.01$ and 1.0. Values of $\rho_{\mathrm{L}} / \rho_{\mathrm{G}}$ in the range 128$\infty$ had little effect on this result, as noted on the plot.
Clearly effects of $\mathrm{Re}$ are small when $\mathrm{Re}>50$, which corresponds to the region where the drag coefficients of spheres and other blunt objects in crossflow are relatively independent of the Reynolds number. For $\operatorname{Re}<50$, however, the resistance of liquid jets in crossflow to deformation, and probably to breakup as well, increases as the drag coefficient increases toward the Stokes flow regime. This is caused by the tendency of increased drag coefficients to increase the rate of relaxation of the liquid jet toward the crossflow velocity. An effect of this nature, however, might very well enhance liquid jet breakup by the liquid column mechanism as discussed by Sallam et al. ${ }^{3}$ this phenomenon clearly merits additional study.

\section{CONCLUSIONS}

The deformation and breakup properties of nonturbulent round liquid jets in gaseous crossflows have been studied computationally and experimentally. Computational conditions included Reynolds numbers of 12.5-1,000, Weber numbers of $0.5-100,000$, Ohnesorge numbers of 0.001-100, liquid/gas density ratios of $2-\infty$ and liquid/gas viscosity ratios of $0.001-1,000$. Experimental test conditions included Reynolds numbers of 345-1100, Weber numbers of 0.6-12, Ohnesorge numbers of 0.003-0.013, liquid/gas density ratios of 806 and 997 and liquid/gas viscosity ratios of 48 and 66. Major conclusions of the study are as follows:

1. Present predictions of the wake and drag properties of solid cylinders in crossflow, and the deformation and deflection properties of round liquid jets in crossflow, were in good agreement with available measurements and numerical predictions.

2. Similar to past experimental observations of the properties of nonturbulent round liquid jets in gaseous crossflows due to Mazallon et al. ${ }^{2}$ and Sallam et al., ${ }^{3}$ remarkable similarities were observed between the deformation and breakup properties of nonturbulent round liquid jets in crossflow and the secondary breakup properties of round liquid drops subjected to shock wave disturbances.

3. The liquid/gas density ratio had a surprisingly small effect on the deformation and breakup of nonturbulent round liquid jets in crossflow for 
values of $\rho_{\mathrm{L}} / \rho_{\mathrm{G}}>30$, particularly when $\mathrm{Oh}$ is small.

4. The Reynolds number of the crossflow had a surprisingly small effect on the deformation and breakup properties of liquid jets for $\operatorname{Re} \geq 50$, where the liquid jet drag coefficients are relatively constant. As the Reynolds number approached the Stokes range, however, liquid jet resistance to deformation and breakup increased significantly due to increased drag coefficients and associated increased jet relaxation toward the crossflow velocity, preventing the jet from reaching the large degrees of deformation associated with breakup. On the other hand, increased drag forces could also contribute to more effective liquid column breakup, an effect that was not studied here but merits attention in the future.

5. A liquid column deformation and breakup regime map, plotted as $\mathrm{We}^{1 / 2} / \mathrm{Oh}$ as a function of 1/Oh, yielded constant deformation and breakup regime boundaries at large $\mathrm{Oh}$, where liquid viscous effects are important, that were relatively independent of other parameters of the flow. Thus, this deformation and breakup regime map is complementary to the classical map of $\mathrm{Hinze}^{23}$ which exhibits similar advantages at small $\mathrm{Oh}$, where surface tension effects are important.

\section{ACKNOWLEDGEMENTS}

This research was sponsored by the U.S. Air Force Office of Scientific Research, Grant. Nos. F49620-99-1-0083 and F49620-02-1-0007 under the Technical Management of J. M. Tishkoff. Assistance from R. Jolly and D. Ma under the University of Michigan Undergraduate Research Opportunity Program is gratefully acknowledged.

\section{REFERENCES}

[1] Vich, G., "Destabilisation d'un Jet Liquide par un Ecoulement Gazeux Perpendiculaire," Ph.D. Dissertation, University of Rouen, Rouen, 1997.

[2] Mazallon, J., Dai, Z., and Faeth, G. M., "Primary Breakup of Nonturbulent Round
Liquid Jets in Gas Crossflows," Atomization and Sprays, Vol. 9, No. 3, 1999, pp. 291-311.

[3] Sallam, K. A., Aalburg, C., and Faeth, G. M., "Breakup of Round Nonturbulent Liquid Jets in Gaseous Crossflows," AIAA J., submitted.

[4] Wu, P.-K., Kirkendall, K.A., Fuller, R.F. and Nejad, A. S., "Breakup Processes of Liquid Jets in Subsonic Crossflows," J. Prop. Power, Vol. 13, No. 1, 1997, pp. 64-73.

[5] Geary, E.L., and Margettes, M. J., "Penetration of a High Velocity Gas Stream by a Water Jet," J. Spacecraft, Vol. 6, No. 1, 1969, pp. 79-81.

[6] Reichenbach, P. R., and Horn, K. P., "Investigation of Injectant Properties in Jet Penetration in a Supersonic Stream," AIAA J., Vol. 9, No. 3, 1971, pp. 469-471.

[7] Kush, E. A., Schetz, J.A., "Liquid Jet Injection into a Supersonic Flow," AIAA J., Vol. 11, No. 9, 1979, pp. 1223-1224.

[8] Schetz, J. A., and Paddye, A., "Penetration of a Liquid Jet in Subsonic Airstreams," AIAA J., Vol. 15, No. 10, 1977, pp. 1385-1390.

[9] Schetz, J.A., Kush, E. A., and Joshi, P. B., "Wave Phenomena in Liquid Jet Breakup in a Supersonic Crossflow," AIAA J., Vol. 15, No. 2, 1979, pp. 774-778.

[10] Nejad, A. S., and Schetz, J. A., "Effects of Properties and Location in the Plume on Droplet Diameter for Injection in a Supersonic Stream," AIAA J., Vol. 21, No. 7, 1983, pp. 956-961.

[11] Nejad, A. S., and Schetz, J. A., "Effects of Viscosity and Surface Tension on a Jet Plume in Supersonic Cross-Flow," AIAA J., Vol. 22, No. 4, 1984, pp. 458-459.

[12] Less, D. M., and Schetz, J. A., "Transient Behavior of Liquid Jets Injected Normal to a High-Velocity Gas Stream," AIAA J., Vol. 24, No. 12, 1986, pp. 1979-1985.

[13] Kitamura, Y., and Takahashi, T., "Stability of a Liquid Jet in Air Flow Normal to the Jet Axis", J. Chemical Engineering of Japan, Vol. 9, No. 4, 1976, pp. 282-286.

[14] Nguyen, T. T., and Karagozian, A. R., "Liquid Fuel Jet in a Subsonic Crossflow," J. Prop. Power, Vol. 8, No. 1, 1992, pp. 21-29.

[15] Karagozian, A.R., "An Analytical Model for the Vorticity Associated with a Transverse Jet," AIAA J., Vol. 24, No. 3, 1986, pp. 429436.

[16] Higuera, F. J., and Martinez, M., "An Incompressible Jet in a Weak Crossflow," J. Fluid Mech., Vol. 249, 1983, pp. 73-97. 
[17] Gel'fand, B.E., Gubin, S.A., and Kogarko, S. M., "Deformation of Liquid Jets and Drops Injected into a Gas Stream," Izvestia Akadamka Nauk. USSR, Mechanic Zhid. i Gaza, Vol. 3, 1970, pp. 82-88.

[18] Borisov, A. A., Gel'fand, B. E., Natanzon, M. S., and Kossov, O. M., "Droplet Breakup Regimes and Criteria for Their Existence," Inzhernerno-Fizicheskii Zhurnal, Vol. 40, 1981, pp. 64-70.

[19] Chou, W.-H., and Faeth, G. M., "Temporal Properties of Secondary Drop Breakup in the Bag Breakup Regime," Int. J. Multiphase Flow, Vol. 24, No. 6, 1998, pp. 889-912.

[20] Chou, W.-H., Hsiang, L.-P., Faeth, G. M., "Temporal Properties of Drop Breakup in the Shear Breakup Regime," Int. J. Multiphase Flow, Vol. 23, No. 4, 1997, pp. 657-669.

[21] Dai, Z., and Faeth, G. M., "Temporal Properties of Secondary Breakup in the Multimode Breakup Regime," Int. J. Multiphase Flow, Vol. 27, No. 2, 2001, pp. 217-236.

[22] Faeth, G. M., Hsiang, L.-P., Wu, P.-K., "Structure and Breakup Properties of Sprays," Int. J. Multiphase Flow, Vol. 1 (supplement), 1005, pp. 99-127.

[23] Hinze, J. O., "Fundamentals of the Hydrodynamic Mechanism of Splitting in Dispersion Processes," Amer. Inst. Chem. Engrs. J., Vol. 1, No. 3, 1955, pp. 289-295.

[24] Hsiang, L.-P., Faeth, G. M., "Near-Limit Drop Deformation and Secondary Breakup," Int. J. Multiphase Flow, Vol. 18, No. 5, 1992, pp. 635-652.

[25] Hsiang, L.-P., Faeth, G. M., "Drop Properties After Secondary Breakup,” Int. J. Multiphase Flow, Vol. 19, No. 5, 1993, pp. 721-735.

[26] Hsiang, L.-P., and Faeth, G. M., "Drop Deformation and Breakup due to Shock Wave and Steady Disturbances," Int. J. Multiphase Flow, Vol. 21, No. 4, 1995, pp. 545-560.

[27] Hwang, S. Z., Liu, Z., and Reitz, R. D., "Breakup Mechanisms and Drag Coefficients of High-Speed Vaporizing Liquid Drops," Atomization and Sprays, Vol. 6, No. 3, 1996, pp. 353-376.

[28] Krzeczkowski, S. A., "Measurement of Liquid Droplet Disintegration Mechanisms," Int. J. Multiphase Flow, Vol. 6, 1980, pp. 227-239.

[29] Ranger, A. A., and Nicholls, J. A., "The Aerodynamic Shattering of Liquid Drops," AIAA J., Vol. 7, No. 2, 1969, pp. 285-290.
[30] Aalburg, C., van Leer, B., Faeth, G. M., "Deformation and Drag Properties of Round Drops Subjected to Shock Wave Disturbances," AIAA J., in press.

[31] Chorin, A. J., "Numerical Solution of the Navier-Stokes Equations," Mathematics of Computation, Vol. 22, No. 104, 1968, pp. 745-762.

[32] Harlow, F. H., and Welch, J. E., "Numerical Calculation of Time-Dependent Viscous Incompressible Flow of Fluid with Free Surface," Phys. Fluids, Vol. 8, No. 12, 1965, pp. 2182-2189.

[33] Sussman, M., Smereka, P., and Osher, S., “A Level Set Approach for Computing Solutions to Incompressible Two-Phase Flow," J. Comp. Phys., Vol. 114, No. 1, 1994, pp. 146-159.

[34] Sussman, M., and Fatemi, E., "An Efficient, Interface Preserving Level Set Redistancing Algorithm and its Application to Interfacial Incompressible Flow," SIAM J. Sci. Computing, Vol. 20, No. 4, 1999, pp. 11651191.

[35] Brackbill, J. U., Kothe, D. B., Zemach, C., “A Continuum Method for Measuring Surface Tension," J. Comp. Phys., Vol. 100, 1991, pp. 335-354.

[36] Coutanceau, M., and Bouard, R., "Experimental Determination of the Main Features of the Viscous Flow in the Wake of a Circular Cylinder in Uniform Translation. Part 2. Unsteady Flow," J. Fluid Mech., Vol. 70, 1977, pp. 257-272.

[37] Kawaguti, P., and Jain, P.," Numerical Study of a Viscous Fluid Flow Past a Circular Cylinder," J. Physical Society of Japan, Vol. 21, 1966, pp. 2055-2062.

[38] Thoman, D. C., and Szewczyk, A. A., "TimeDependent Viscous Flow over a Circular Cylinder," Phys. Fluids Supplement, Vol. 12, 1969, pp. II76.

[39] Kovasznay, L. S. G., "Hot-Wire Investigation of the Wake Behind Cylinders at Low Reynolds Numbers," Proc. Roy. Soc. (London), Vol. 198(A), 1948, pp. 174-180.

[40] Roshko, A., "On the Drag and Shedding Frequency of Two-Dimensional Bluff Bodies," NACA Technical Note 3169, Washington, D.C., 1954.

[41] Berger, E., and Wille, R., "Periodic Flow Phenomena," Ann. Rev. Fluid Mech., Vol. 4, 1972, pp. 313-340.

[42] Nishioka, M., and Sato, M., "Mechanism of Determination of the Shedding Frequency of 
Vortices Behind a Cylinder at Low Reynolds Numbers," J. Fluid Mech., Vol. 89, 1978, pp. 49-60.

[43] Wieselsberger, C., "Ergebnisse der Aerodynamischen Versuchsanstalt zu Göttingen,” R. O. Oldenbourg, Munich, 1927.

[44] Tritton, D. J., "Experiments on the Flow Past a Cylinder at Low Reynolds Numbers," $J$. Fluid Mech., Vol. 6, 1959, pp. 547-567.

[45] Nishioka, M., "Hot-Wire Investigation of the Steady Laminar Wake Behind a Thin Flat Plate Placed Perpendicularly to a Uniform Flow," Bulletin of the University of Osaka, Prefect, Series A, Vol. 21, 1972, pp. 227-232.

[46] Takami, H., and Keller, H. B., "Steady TwoDimensional Viscous Flow of an Incompressible Fluid Past a Circular Cylinder," Phys. Fluids, Vol. 12(II), 1969, pp. 51-56.

[47] Dennis, S. C. R., and Chang, G.-Z., "Numerical Simulation for Steady Flow Past a Circular Cylinder at Reynolds Numbers up to 100," J. Fluid Mech., Vol. 42, 1970, pp. 471489.
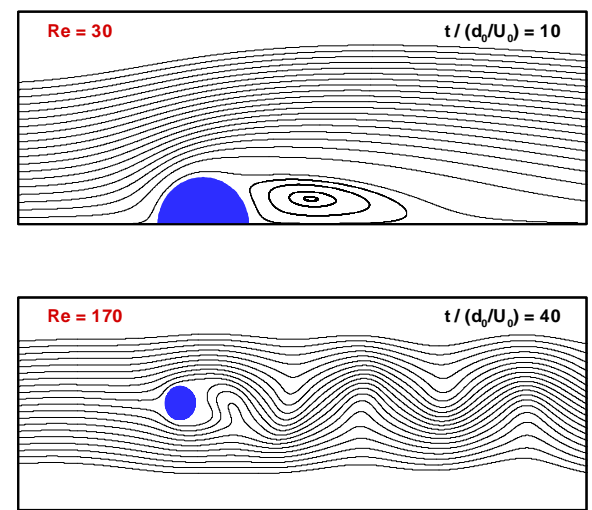

Fig. 1 Streamline patterns for a stable wake $(\operatorname{Re}=30)$ and for eddy shedding $(\operatorname{Re}=170)$ behind a solid circular cylinder in crossflow

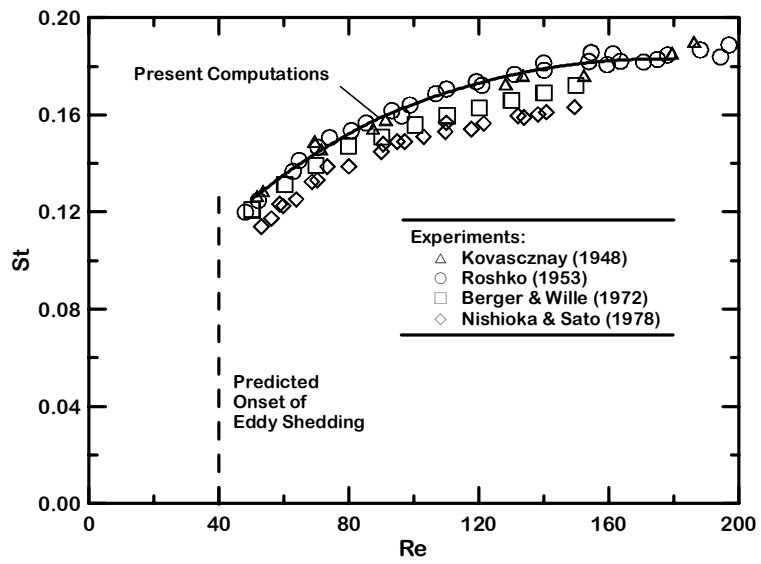

Fig. 2 Strouhal numbers of the eddy shedding frequency as a function of the Reynolds number for solid circular cylinders in crossflow.

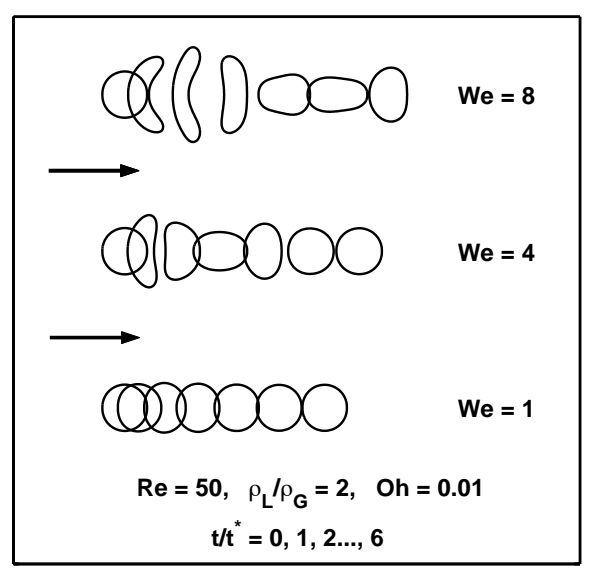

Fig. 3 Visualization of liquid crossections as a function of time for various Weber numbers $(\operatorname{Re}=50$, $\left.\rho_{\mathrm{L}} / \rho_{\mathrm{G}}=2, \mathrm{Oh}=0.01 ; \mathrm{t} / \mathrm{t}^{*}=0,1,2, \ldots, 6\right)$.

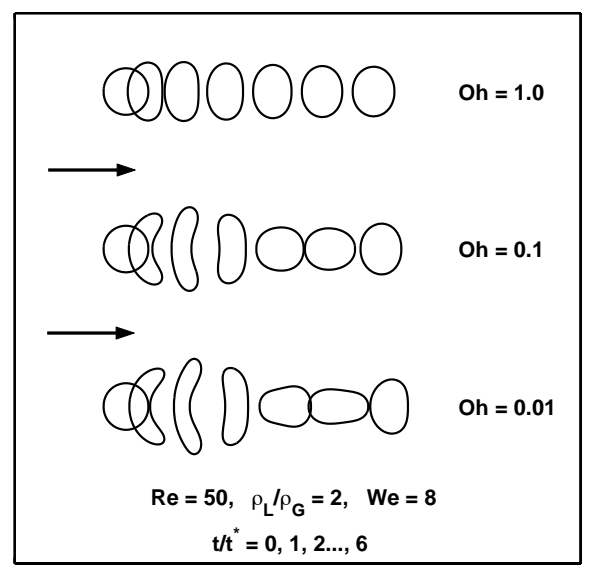

Fig. 4 Visualization of liquid crossections as a function of time for various Ohensorge numbers $\left(\mathrm{Re}=50, \rho_{\mathrm{L}} / \rho_{\mathrm{G}}=2, \mathrm{We}=32 ; \mathrm{t} / \mathrm{t}^{*}=0,1,2, \ldots, 6\right)$. 


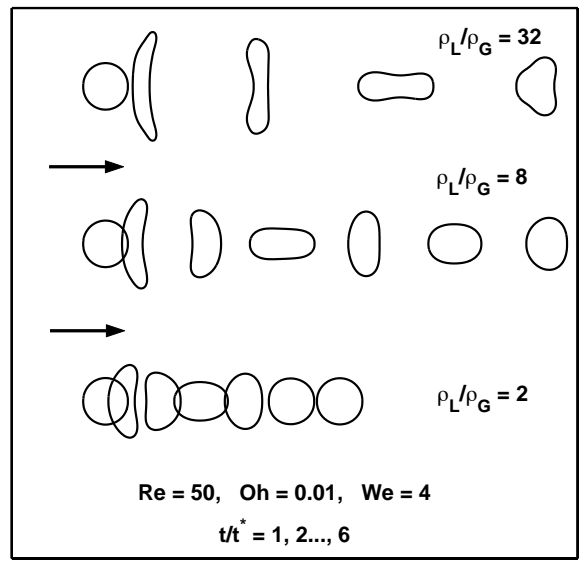

Fig. 5 Visualization of liquid crossections as a function of time for various liquid/gas density ratios $\left(\mathrm{Re}=50, \mathrm{Oh}=0.01, \mathrm{We}=32 ; \mathrm{t} / \mathrm{t}^{*}=0,1,2, \ldots, 6\right)$.
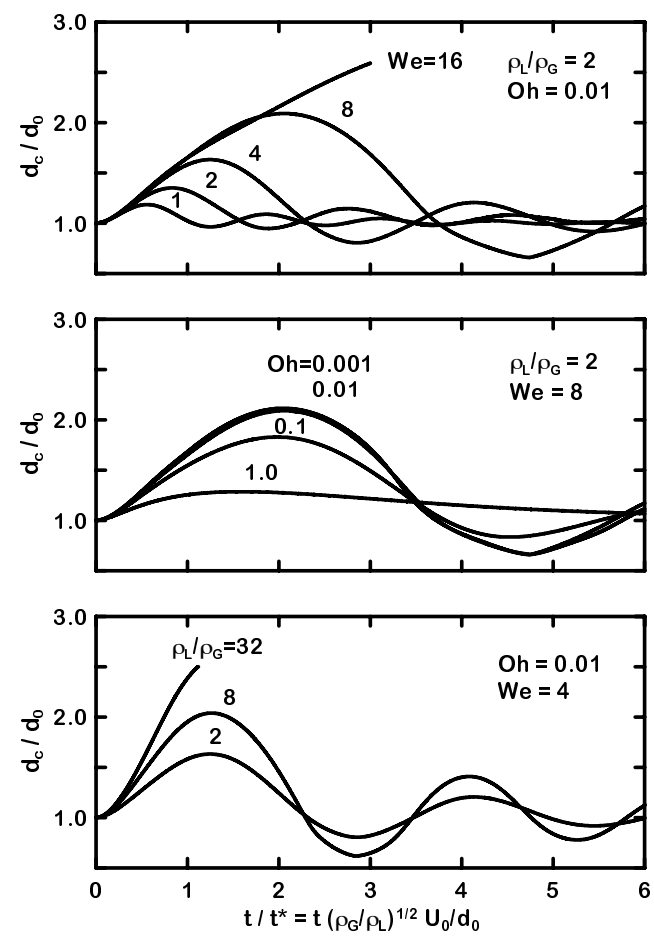

Fig. 6 Liquid jet crosstream deformation, $\mathrm{d}_{\mathrm{c}} / \mathrm{d}_{0}$, as a function of time for various Weber numbers, Ohnesorge numbers and liquid/gas density ratios.
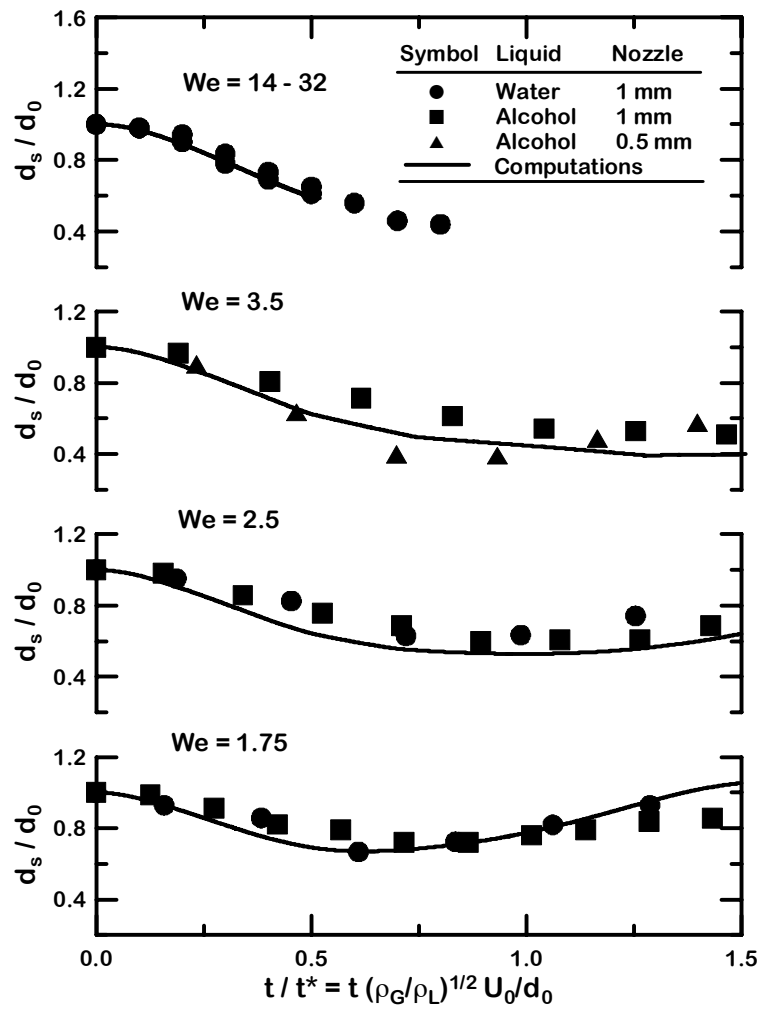

Fig. 7 Measured and computed streamwise liquid column dimensions, $d_{s} / d_{0}$, as a function of time for various Weber numbers.

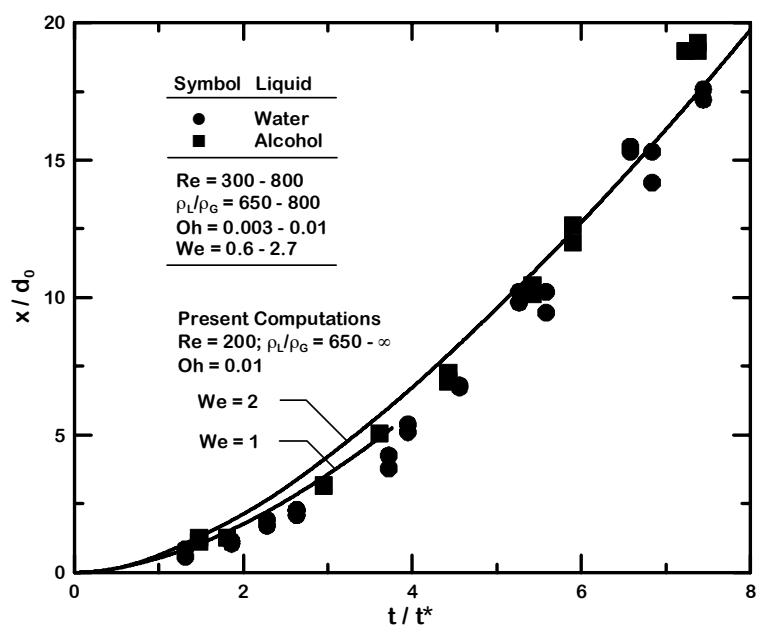

Fig. 8 Streamwise deflection of liquid jet trajectories as a function of time. 


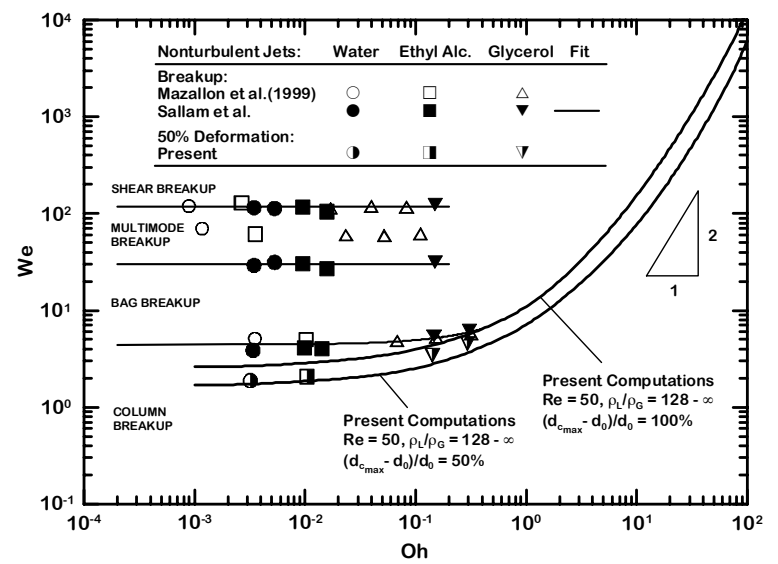

Fig. 9 Predicted and measured liquid jet breakup regime map in the classical drag-force/surfacetension-force (We) and viscous-force/surfacetension-force (Oh) coordinates of Hinze [23].

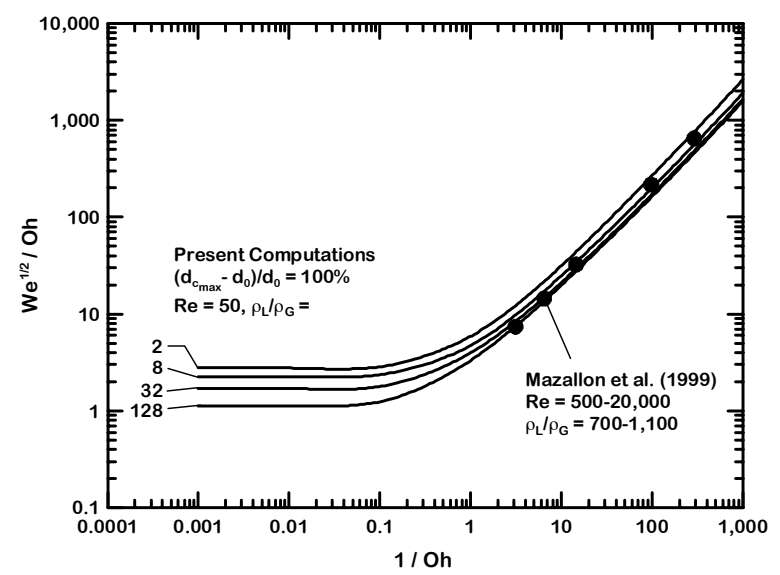

Fig. 10 Predicted and measured liquid jet breakup regime map in drag-force/liquid-viscous-force $\left(\mathrm{We}^{1 / 2} / \mathrm{Oh}\right)$ and surface-tension-force/liquid-viscousforce $(1 / \mathrm{Oh})$ coordinates.

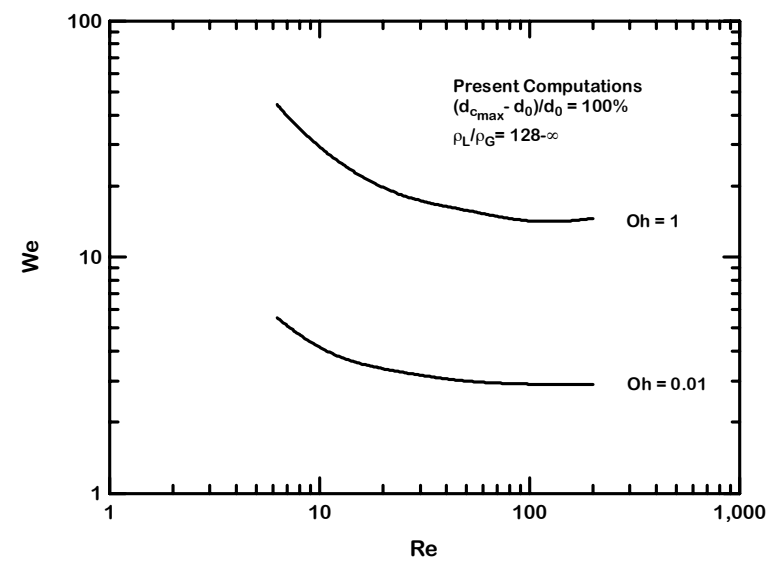

Fig. 11 Effect of Reynolds number on the We for $100 \%$ deformation of liquid jets in crossflow for various Ohnesorge numbers. 